\title{
Calculation of the optimal imaging parameters for frequency modulation atomic force microscopy
}

\author{
Franz J. Giessibl * , Hartmut Bielefeldt, Stefan Hembacher, Jochen Mannhart \\ Universität Augsburg, Institute of Physics, Electronic Correlations and Magnetism, Experimentalphysik VI, Universitätsstrasse 1, D-86135 \\ Augsburg, Germany
}

Received 1 July 1998; accepted 11 August 1998

\begin{abstract}
True atomic resolution of conductors and insulators is now routinely obtained in vacuum by frequency modulation atomic force microscopy. So far, the imaging parameters (i.e., eigenfrequency, stiffness and oscillation amplitude of the cantilever, frequency shift) which result in optimal spatial resolution for a given cantilever and sample have been found empirically. Here, we calculate the optimal set of parameters from first principles as a function of the tip-sample system. The result shows that the either the acquisition rate or the signal-to-noise ratio could be increased by up to two orders of magnitude by using stiffer cantilevers and smaller amplitudes than are in use today. (c) 1999 Elsevier Science B.V. All rights reserved.
\end{abstract}

PACS: 07.79 Lh; 61.16 Ch; 87.64 Dz; $34.20 \mathrm{Cf}$

Keywords: Atomic force microscopy; Frequency modulation atomic force microscopy; Dynamic force microscopy; Atomic resolution; Tip-sample interaction; Dissipation; Thermal noise

\section{Introduction}

Although Binnig, Quate and Gerber anticipated that the atomic force microscope (AFM) they invented in 1985 [1] would ultimately achieve true atomic resolution in vacuum, it took almost 10 years before the $\mathrm{Si}(111)-(7 \times 7)$ reconstruction $[2-7]$ and other conducting [8] and insulating surfaces $[9,10]$ were imaged with atomic resolution by AFM. Frequency modulation (FM) AFM, originally invented by Albrecht et al. in 1991 [11] for magnetic force

\footnotetext{
* Corresponding author. Tel.: +49-821-598-3675; Fax: +49821-598-3652; E-mail: franz.giessibl@physik.uni-augsburg.de
}

microscopy, is the method which was used in the first demonstration of true atomic resolution in vacuum and is now common practice in vacuum AFM. In this technique, a cantilever (CL) with spring constant $k$ and eigenfrequency $f_{0}$ is subject to positive feedback such that it oscillates with a constant amplitude $A_{0}$. When the oscillating CL is approached to a sample, its oscillation frequency changes from $f_{0}$ to $f=f_{0}+\Delta f$ due to the forces between the tip of the CL and the sample. Scanning the CL across the sample ( $x-y$ plane) and adjusting $z$ such that $f$ is constant yields a map $z(x, y, \Delta f, k$, $\left.f_{0}, A_{0}\right)$. This map provides an atomic picture of the surface if the vertical noise is smaller than the atomic corrugation. Up to now, combinations of $\Delta f$, 
Table 1

Parameters used for imaging various surfaces by FM-AFM newline

\begin{tabular}{lccccclcl}
\hline$k[\mathrm{~N} / \mathrm{m}]$ & $f_{0}[\mathrm{kHz}]$ & $\Delta f[\mathrm{~Hz}]$ & $A_{0}[\mathrm{~nm}]$ & $k A_{0}[\mathrm{nN}]$ & $\gamma^{a}[\mathrm{fN} \sqrt{\mathrm{m}}]$ & $\mathrm{Sample}$ & $\begin{array}{l}\text { Ref. } \\
\text { Lateral } \\
\text { resolution }\end{array}$ \\
\hline 43.0 & 276.0 & -60 & 40.0 & 1720 & -74.7 & $\mathrm{Si}(111)$ & {$[3]$} & atomic \\
17.0 & 114.0 & -70 & 32.0 & 544 & -65.4 & $\mathrm{Si}(111)$ & {$[2]$} & atomic \\
23.5 & 153.0 & -70 & 19.0 & 447 & -28.2 & $\mathrm{Si}(111)$ & {$[6]$} & atomic \\
33.0 & 264.0 & -670 & 4.0 & 132 & -21.2 & $\mathrm{Si}(001)$ & {$[7]$} & atomic \\
30.0 & 168.0 & -80 & 13.0 & 390 & -21.2 & $\mathrm{NaCl}(001)$ & {$[10]$} & atomic \\
36.0 & 160.0 & -63 & 12.7 & 457 & -20.3 & $\mathrm{InAs}(110)$ & {$[12]$} & atomic \\
28.0 & 270.0 & -80 & 15 & 420 & -15.2 & $\mathrm{TiO}(110)$ & {$[13]$} & atomic \\
37.0 & 276.0 & -50 & 10.0 & 370 & -6.7 & $\mathrm{Si}(111)$ & {$[7]$} & atomic \\
41.0 & 172.0 & -10 & 16.0 & 654 & -4.8 & $\mathrm{Si}(111)$ & {$[14]$} & atomic \\
34.0 & 151.0 & -6 & 20.0 & 680 & -3.8 & $\mathrm{InP}(110)$ & {$[8]$} & atomic \\
10.0 & 290.0 & -95 & 10.0 & 100 & -3.3 & $\mathrm{Si}(111)$ & {$[5]$} & atomic \\
37.0 & 276.0 & -350 & 1.5 & 55.5 & -2.7 & $\mathrm{Si}(111)$ & {$[7]$} & atomic \\
2.5 & 60.0 & -16 & 15.0 & 37.5 & -1.2 & $\mathrm{KCl}(001)$ & {$[15]$} & $\approx 3 \mathrm{~nm}$ \\
2.5 & 60.0 & -32 & 3.3 & 8.25 & -0.3 & $\mathrm{Si}(111)$ & {$[15]$} & $\approx 0.6 \mathrm{~nm}(x), 2 \mathrm{~nm}(y)$ \\
\hline
\end{tabular}

${ }^{\mathrm{a}} \gamma=\Delta f k A_{0}^{3 / 2} / f_{0}$, see Ref. [16].

$k, f_{0}$, and $A_{0}$ which do provide true atomic resolution have been found empirically. With currently available CLs, the oscillation amplitude $A_{0}$ needs to be up to 100 times the interatomic distances for obtaining atomic resolution (see Table 1). However, the spring constant $k$ and the eigenfrequency $f_{0}$ have to be selected from the discrete set of commercially available CLs. Since the spring constant of a CL cannot be freely chosen, it is important to clarify for which set of operating parameters $\left(k, f_{0}, A_{0}\right)$ best performance is to be expected. Here, we present a calculation based on first principles for the optimal set of $k, f_{0}$ and $A_{0}$ as a function of the tip-sample potential $V_{\mathrm{ts}}(x, y, z)$. The models for $V_{\mathrm{ts}}(x, y, z)$ we use here include exponential and inverse-power laws.

\section{Calculation of vertical noise}

Since the imaging signal in FM-AFM is a frequency shift $\Delta f$, the best signal-to-noise ratio is expected for large $\Delta f$ and little noise $\delta(\Delta f)$. However, for a given minimum distance $d$ between the front atom of the CL and the sample both $\Delta f$ and $\delta(\Delta f)$ decrease with amplitude. If $\Delta f$ were independent of $A_{0}$, minimal noise would result for $A_{0} \rightarrow \infty$. Since the magnitude of $\Delta f$ decreases with ampli- tude, it is not straightforward to see whether there is an optimum (minimum in vertical noise) for a specific set of $k, f_{0}$ and $A_{0}$. These optimal parameters will depend on the characteristics of the tip-sample potential.

Three major contributors to the vertical noise in $z\left(x, y, \Delta f, k, f_{0}, A_{0}\right)$ can be identified (see Fig. 1).

(1) Frequency noise: a variation $\delta d$ leads to a variation in the frequency shift according to $\delta(\Delta f)$

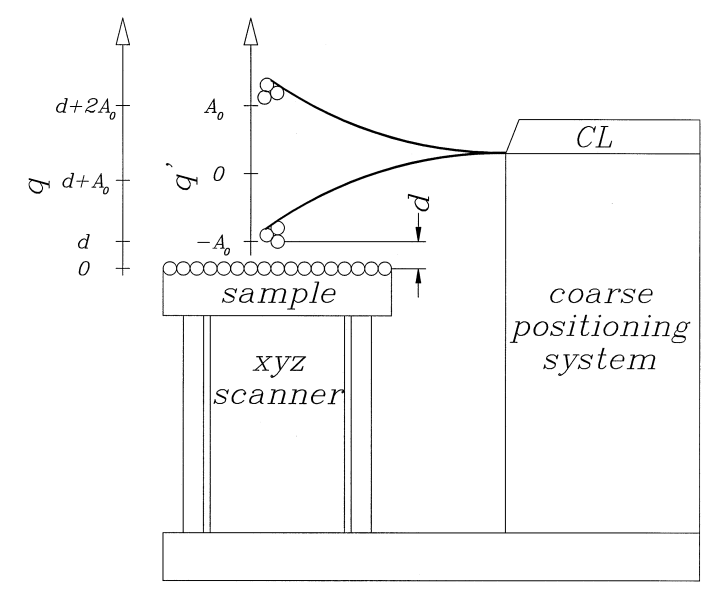

Fig. 1. Schematic of a frequency modulation atomic force microscope. 
$=(\partial(\Delta f)) /(\partial d) \delta d$. Thus, the vertical noise caused by frequency noise is:

$$
\delta z_{f}=\frac{\delta(\Delta f)}{\frac{\partial(\Delta f)}{\partial d}} .
$$

Albrecht et al. [11] have calculated the thermodynamic lower limit of the frequency noise

$\delta f_{\text {thermal }}=\delta(\Delta f)_{\text {thermal }}=\sqrt{\frac{k_{\mathrm{B}} T B f_{0}}{k A_{0}^{2} \pi Q_{0}}}$

where $k_{\mathrm{B}}$ is the Boltzmann constant, $T$ the absolute temperature, $B$ the detection bandwidth and $Q_{0}$ the quality factor of the CL [11]. The detection bandwidth $B$ determines the imaging speed. If a section with a width of 100 atoms is to be imaged at an imaging speed of three lines per second, $B$ needs to be at least $2 \times 100 \times 3 / \mathrm{s}=600 \mathrm{~Hz}$.

There is also a contribution to frequency noise $\delta(\Delta f)_{\text {instrumental }}$ which depends on the quality of the frequency detector. Since these noise sources are uncorrelated, the total frequency noise is given by

$\delta(\Delta f)=\sqrt{\delta(\Delta f)_{\text {thermal }}^{2}+\delta(\Delta f)_{\text {instrumental }}^{2}}$.

The denominator in Eq. $(1),(\partial(\Delta f)) /(\partial d)$, is calculated further below.

(2) Amplitude noise: the fluctuation of the total energy of the CL is approximately $k_{\mathrm{B}} T$. Thus, $k\left(A_{0}\right.$ $\left.+\delta A_{0}\right)^{2} / 2 \approx k A_{0}^{2} / 2+k_{\mathrm{B}} T$ and the amplitude noise is given by:

$\delta A_{0} \approx \frac{k_{\mathrm{B}} T}{k A_{0}}$.

For typical values of $k A_{0}$ (Table 1), this contribution is negligible even at room temperature.

(3) Mechanical noise: the mechanical loop from CL to sample is closed by the xyz scanner and the coarse positioning system. Acoustic noise, building vibrations etc. cause a variation of the distance between $C L$ and sample $\delta Z_{\text {instr: }}$ Proper design of the microscope and insulation from external vibrations help to bring $\delta Z_{\text {instr. }}$ down to a few picometers.
Since the noise contributions in $z$-direction are statistically independent, the total $z$-noise is:

$$
(\delta z)^{2}=\left(\frac{\delta(\Delta f)}{\frac{\partial(\Delta f)}{\partial d}}\right)^{2}+\left(\delta A_{0}\right)^{2}+\left(\delta Z_{\text {instr. }}\right)^{2} .
$$

Since amplitude- and mechanical noise are negligible, in the following, we only consider the noise contribution from frequency noise.

\subsection{Calculation of the derivative of the frequency shift}

\subsubsection{Frequency shift for inverse-power forces}

For tip-sample forces given by

$F_{\mathrm{ts}}(q)=\frac{C}{q^{n}}$

where $C$ is a constant and $q$ is the distance between the center of the front atom of the tip and the plane defined by the centers of the surface atoms, the frequency shift $\Delta f=f-f_{0}$ is given by Ref. [16]:

$\Delta f_{s A_{0}}=n \frac{f_{0}}{2 k} \frac{C}{d^{n+1}}$

if $A_{0} \ll d$. For $A_{0} \gg d$ the frequency shift is given by:

$\Delta f_{l A_{0}}=Y_{n} \frac{f_{0}}{k A_{0}^{3 / 2}} \frac{C}{d^{n-1 / 2}}$

with

$Y_{n}:=\frac{1}{\sqrt{2 \pi}} \int_{-\infty}^{\infty} \frac{1}{\left(1+y^{2}\right)^{n}} \mathrm{~d} y$.

Pertinent values of $Y_{n}$ are $Y_{1}=1 / \sqrt{2}, Y_{2} \approx 0.36$, $Y_{3} \approx 0.27, Y_{4} \approx 0.22, Y_{7} \approx 0.16$ and $Y_{13} \approx 0.11$ [16].

For functions $g_{s}(d)=c d^{-3 / 2}$ and $g_{l}\left(A_{0}\right)=$ $A_{0}^{-3 / 2}$ a function

$g\left(d, A_{0}\right)=\frac{1}{\left[c^{-m} d^{3 m / 2}+A_{0}^{3 m / 2}\right]^{1 / m}}$

fulfills $\lim _{A_{0} / d \rightarrow 0} g\left(d, A_{0}\right)=g_{s}(d)$ and $\lim _{A_{0} / d \rightarrow \infty}$ $g\left(d, A_{0}\right)=g_{l}\left(A_{0}\right)$. The simplest case $m=1$ yields 
an approximation for $\Delta f$ which is sufficiently precise for intermediate amplitudes ${ }^{1}$

$$
\begin{aligned}
\Delta f & =\frac{Y_{n} f_{0}}{k\left(A_{0}^{3 / 2}+\frac{2 Y_{n}}{n} d^{3 / 2}\right)} \frac{C}{d^{n-1 / 2}} \\
& =\frac{Y_{n} f_{0} C}{k} \frac{1}{A_{0}^{3 / 2} d^{n-1 / 2}+\frac{2 Y_{n}}{n} d^{n+1}} .
\end{aligned}
$$

The variation of the frequency shift with distance is given by the derivative:

$$
\begin{aligned}
\frac{\partial(\Delta f)}{\partial d} & \\
= & -\frac{Y_{n} f_{0} C}{k} \\
& \times \frac{(n-1 / 2) A_{0}^{3 / 2} d^{n-3 / 2}+2 Y_{n}(1+1 / n) d^{n}}{\left(A_{0}^{3 / 2} d^{n-1 / 2}+\frac{2 Y_{n}}{n} d^{n+1}\right)^{2}} .
\end{aligned}
$$

\subsubsection{Frequency shift for exponential forces}

For exponential forces

$F_{\text {ts }}(q)=F_{0} \mathrm{e}^{-q / \lambda}$

the frequency shift for small amplitudes $A_{0} \ll \lambda$ is:

$\Delta f_{s A_{0}}=\frac{f_{0}}{2 k \lambda} F_{0} \mathrm{e}^{-d / \lambda}$

and the frequency shift for large amplitudes $A_{0} \gg \lambda$ [16]:

$\Delta f_{l A_{0}}=\frac{f_{0} \sqrt{\lambda}}{k A_{0}^{3 / 2}} \frac{F_{0} \mathrm{e}^{-d / \lambda}}{\sqrt{2 \pi}}$.

Again, we can create an approximation for all amplitudes

$\Delta f=\frac{f_{0}}{2 k \lambda\left(1+\sqrt{\frac{\pi}{2}}\left(\frac{A_{0}}{\lambda}\right)^{3 / 2}\right)} F_{0} \mathrm{e}^{-d / \lambda}$

\footnotetext{
${ }^{1}$ For amplitudes in the order of the decay length $\left(A_{0}=d, \lambda\right)$, the error of the all-amplitude approximation is $7 \%, 2 \%, 4 \%$ and $90 \%$ for exponential forces and inverse-power forces $n=13,7$ and 1, respectively. A precise formula for $n=1$ is given in $\mathrm{H}$. Hölscher et al. [18].
}

with the derivative

$$
\frac{\partial(\Delta f)}{\partial d}=-\frac{1}{\lambda} \Delta f \text {. }
$$

With this calculation $(\partial(\Delta f)) /(\partial d)$ all the elements of the $z$-noise are complete. Finally, we have to take into account that we cannot choose the stiffness of the CL $k$ independently from the amplitude $A_{0}$. Forces between tip and sample in vacuum are in general attractive before the CL makes contact. Therefore, there is a distance region where the CL is unstable and snaps uncontrolled to the surface ('jump-to-contact'), unless either $k>\max$ $\left(-\left(\partial^{2} V_{\mathrm{ts}}\right) /\left(\partial d^{2}\right)\right)=k_{\mathrm{ts}}^{\max }$ [17] or $k A_{0}>\max \left(-F_{\mathrm{ts}}\right)$ $=F_{\mathrm{ts}}^{\max }[16]$.

These two conditions can be merged to

$k=s \frac{F_{\mathrm{ts}}^{\max }}{A_{0}+F_{\mathrm{ts}}^{\max } / k_{\mathrm{ts}}^{\max }}$

with a 'safety factor' $s>1$.

\subsection{Effect of nonconservative $V_{t s}$}

The tip-sample potential has so far been treated as nondissipative. However, Erlandsson et al. [4], Bammerlin et al. [10], Sugawara et al. [14], Guethner [5] and others have found significant damping of the $\mathrm{CL}$ when it oscillates close to the surface. A general model in analogy to friction is that the dissipative force component is given by

$F_{\text {diss }}(q)=-\mu F_{\text {ts }}(q) \frac{\frac{\mathrm{d} q}{\mathrm{~d} t}}{\left|\frac{\mathrm{d} q}{\mathrm{~d} t}\right|}\left|\frac{\mathrm{d} q}{\mathrm{~d} t}\right|^{r}$.

The parameter $r$ determines the type of 'friction': $r=0$ corresponds to velocity-independent friction, $r=1$ is commonly used for treating damped oscillators and $r=2$ describes friction in fluids. Unfortunately, little is known about the nature of the dissipation process in FM-AFM. For the simplest case $r=0$, the energy loss per oscillation cycle $\Delta E_{\mathrm{ts}}$ is given by:

$$
\Delta E_{\mathrm{ts}}=-\int_{d+2 A_{0}}^{d} \mu F_{\mathrm{ts}}(q) \mathrm{d} q+\int_{d}^{d+2 A_{0}} \mu F_{\mathrm{ts}}(q) \mathrm{d} q .
$$




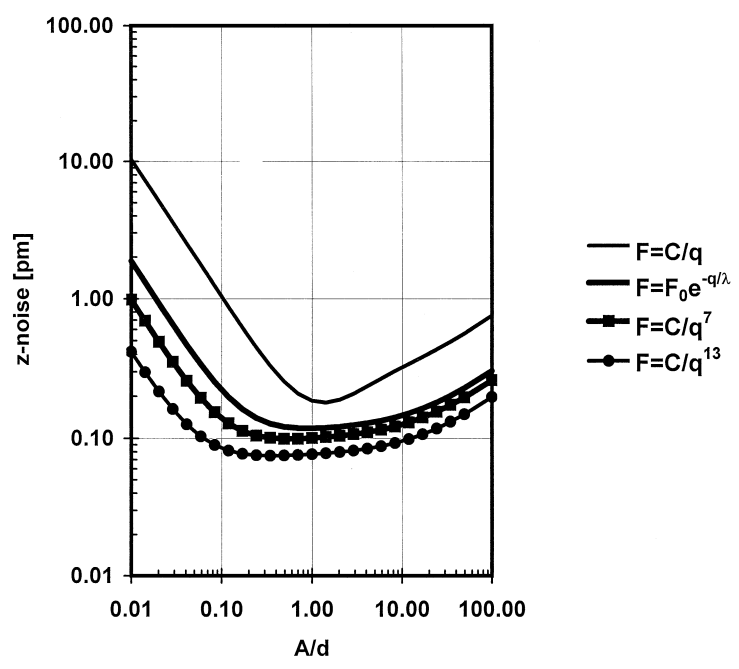

Fig. 2. Calculated noise in sample topography (perpendicular to surface) as a function of amplitude for exponential and inversepower force models.

Since $\int F_{\mathrm{ts}} \mathrm{d} q=V_{\mathrm{ts}}$,

$\Delta E_{\mathrm{ts}}=2 \mu\left[V_{\mathrm{ts}}\left(d+2 A_{0}\right)-V_{\mathrm{ts}}(d)\right]$.

This energy loss diminishes the quality factor $Q_{0}$ of the CL. Since $Q_{0}=E / \Delta E_{\mathrm{CL}}$, where $E=k A_{0}^{2} / 2$ is the total energy in the CL and $\Delta E_{\mathrm{CL}}$ is the energy loss due to internal friction in the CL, the effective $Q$-value is given by:

$Q=\frac{E}{\Delta E_{\mathrm{CL}}+\Delta E_{\mathrm{ts}}}=\frac{Q_{0}}{1+2 Q_{0} \Delta E_{\mathrm{ts}} / k A_{0}^{2}}$.

The negative effect of dissipation on the $Q$ factor is partially compensated with using large amplitudes. Bammerlin et al. [10] have measured the dissipation for a silicon tip interacting with potassium chloride. Analysis of their data yields $\mu \approx 0.05$.

\section{Results and conclusion}

The $z$-noise is thus given by

$$
\begin{aligned}
\delta z_{f}= & \frac{k}{Y_{n} F_{\mathrm{ts}}^{\max }} \frac{d^{2}\left(\left(\frac{A_{0}}{d}\right)^{3 / 2}+\frac{2 Y_{n}}{n}\right)^{2}}{\left(n-\frac{1}{2}\right)\left(\frac{A_{0}}{d}\right)^{3 / 2}+2 Y_{n}(1+1 / n)} \\
& \times \sqrt{\frac{k_{\mathrm{B}} T B}{\pi Q k A_{0}^{2} f_{0}}}
\end{aligned}
$$

for inverse-power forces and

$$
\delta z_{f}=\frac{2 k \lambda^{2}}{F_{\mathrm{ts}}^{\max }}\left(1+\sqrt{\frac{\pi}{2}}\left(\frac{A_{0}}{\lambda}\right)^{3 / 2}\right) \sqrt{\frac{k_{\mathrm{B}} T B}{\pi Q k A_{0}^{2} f_{0}}}
$$

for exponential forces. In both cases, the noise is proportional to $1 / F_{\mathrm{ts}}^{\max }$, i.e., the greater the maximum attractive force, the easier it is to obtain true atomic resolution. Also, the noise is proportional to $1 / \sqrt{f_{0}}$, i.e., the higher the frequency, the lower the noise. The most interesting implications of Eqs. (23) and (24) are the dependence of noise with amplitude. The stiffness of the CL is calculated with Eq. (18). The safety factor $s$ is set to 100 (most authors use $s \gg 1$ see column ' $k A_{0}$ ' in Table 1 ).

Fig. 2 shows a plot of $\delta z_{f}$ for inverse-power forces with $n=1,7$ and 13 and exponential forces with $F_{\mathrm{ts}}^{\max }=3 \mathrm{nN}, d=250 \mathrm{pm}$ and $\lambda=a_{\mathrm{Bohr}}=52.9$ pm as a function of $A_{0} / d$. The corresponding force models are listed in Table 2. The properties of the CL in use are $Q_{0}=10000, f_{0}=100 \mathrm{kHz}, T=300$ $\mathrm{K}$, the frequency detector is set to $B=1 \mathrm{kHz}$ and its electronic noise is $\delta(\Delta f)_{\text {instrumental }}=0.1 \mathrm{~Hz}$. For $F_{\text {ts }}$

Table 2

Models used for tip-sample potential

\begin{tabular}{llllll}
\hline Force model & $V_{\mathrm{ts}}(q)$ & $F_{\mathrm{ts}}(q)$ & $F_{\mathrm{ts}}^{\max }$ & $k_{\mathrm{ts}}^{\max }$ & $\Delta E_{\mathrm{ts}}$ \\
\hline Inverse-power, $n>1$ & $\frac{C}{n-1} \frac{1}{q^{n}-1}$ & $C / q^{n}$ & $C / d^{n}$ & $n F_{\mathrm{ts}}^{\max } / d$ & $\mu \frac{F_{\mathrm{ts}}^{\max } d}{n-1}\left[1-\frac{1}{\left(1+2 A_{0} / d\right)^{n-1}}\right]$ \\
Inverse-power, $n=1$ & $C \ln (q)$ & $C / q$ & $C / d$ & $F_{\mathrm{ts}}^{\max } / d$ & $\mu F_{\mathrm{ts}}^{\max } d \ln \left(1+2 A_{0} / d\right)$ \\
Exponential & $F_{0} \lambda \mathrm{e}^{-q / \lambda}$ & $F_{0} \mathrm{e}^{-q / \lambda}$ & $F_{0} \mathrm{e}^{-d / \lambda}$ & $F_{\mathrm{ts}}^{\max } / \lambda$ & $\mu F_{\mathrm{ts}}^{\max _{2}} \lambda\left(1-\exp ^{-2 A_{0} / \lambda}\right)$ \\
\hline
\end{tabular}




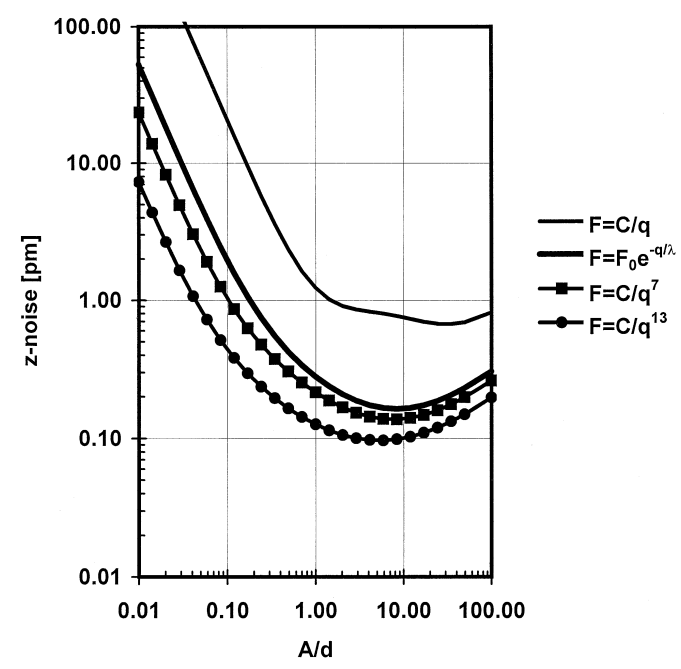

Fig. 3. Calculated noise in sample topography as a function of amplitude for exponential and inverse-power force models with dissipation.

$=C / q$, the optimum is reached for $A_{0} / d \approx 1$. The corresponding spring constant is $k=500 \mathrm{~N} / \mathrm{m}$. For forces with shorter ranges $(n=7,13$ and exponential), the lowest noise is obtained for even smaller amplitudes. The corresponding optimal spring constants range up to $3000 \mathrm{~N} / \mathrm{m}$.

Fig. 3 shows the noise for nonconservative potentials. For each cycle, an energy $\Delta E_{\mathrm{ts}}$ is dissipated with $\delta E_{\mathrm{ts}}=2 \mu\left(V_{\mathrm{ts}}\left(d+2 A_{0}\right)-V_{\mathrm{ts}}(d)\right)$ with $\mu=$ 0.05 . As expected, larger amplitudes are required for minimum noise. The minimal noise is less pronounced than in the case without damping. The corresponding optimal stiffness is between 30 and $200 \mathrm{~N} / \mathrm{m}$.

In summary, we have shown why CLs with $k \approx 10$ $\mathrm{N} / \mathrm{m}$ require amplitudes in the order of $10 \mathrm{~nm}$. If CLs with $k \approx 300 \mathrm{~N} / \mathrm{m}$ were available, $B$ could be increased by 100 or the vertical noise would drop by a factor of 10 . Since the $z$-noise level is already comparable to the other noise sources $\delta A_{0}$ and $\delta Z_{\text {instr, }}$, the major practical advantage is an increase in bandwidth which would allow faster scanning.

By comparing Figs. 2 and 3 it is clear that dissipation is crucial for determining the optimal operating amplitude-zero dissipation would favor stiff cantilevers and strong dissipation requires large amplitudes and softer cantilevers. More work on the investigation of the dissipation process between tip and sample needs to be done for improving the performance of FM-atomic force microscopy. Also, Eqs. (23) and (24) suggest that using CLs with higher $f_{0}$ results in less noise. However, if the dissipative part of the tip-sample force depends on the relative velocity between tip and sample $(r \geq 1$ in Eq. (19)), using CLs with higher frequencies might result in poorer resolution, since the dissipated energy will be proportional to $f_{0}$.

\section{Acknowledgements}

We thank Lukas Howald for discussions. This work was supported by BMBF Grant 13N6918/1.

\section{References}

[1] G. Binnig, C.F. Quate, Ch. Gerber, Phys. Rev. Lett. 56 (1986) 930

[2] F.J. Giessibl, Science 267 (1995) 68.

[3] S. Kitamura, M. Iwatsuki, Jpn. J. Appl. Phys. 34 (1995) L145.

[4] R. Erlandsson, L. Olsson, P. Martensson, Phys. Rev. B 54 (1996) R8309.

[5] P. Güthner, J. Vac. Sci. Technol. B 14 (1996) 2428.

[6] R. Lüthi, E. Meyer, M. Bammerlin, A. Baratoff, T. Lehmann, L. Howald, Ch. Gerber, H.J. Guntherodt, Z. Phys. B 100 (1996) 165.

[7] S. Kitamura, M. Iwatsuki, Jpn. J. Appl. Phys. 35 (1996) L668.

[8] Y. Sugawara, M. Ohta, H. Ueyama, S. Morita, Science 270 (1995) 1648.

[9] J. Patrin, presentation at STM 95, Aspen, CO, 1995.

[10] M. Bammerlin, R. Lüthi, E. Meyer, A. Baratoff, J. Lü, M. Guggisberg, Ch. Gerber, L. Howald, H.J. Güntherodt, Probe Microsc. 1 (1997) 3.

[11] T.R. Albrecht, P. Grütter, D. Horne, D. Rugar, J. Appl. Phys. 69 (1991) 668.

[12] W. Allers, A. Schwarz, U.D. Schwarz, R. Wiesendanger, Rev. Sci. Instr. 69 (1998) 221.

[13] K. Fukui, H. Onishi, Y. Iwasawa, Phys. Rev. Lett. 79 (1997) 4202.

[14] Y. Sugawara, H. Ueyama, T. Uchihashi, M. Ohta, Y. Yanase, T. Shigematsu, M. Suzuki, S. Morita, Materials Research Society 1996 Fall Meeting, in: J. Michel, T. Kennedy, K. Wada, K. Thonke (Eds.), Proceedings E: Defects in Electric Materials II, Boston, December 1996, p. 16.

[15] F.J. Giessibl, Jpn. J. Appl. Phys. 33 (1994) 3726.

[16] F.J. Giessibl, Phys. Rev. B 56 (1997) 16010.

[17] N. Burnham, R.J. Colton, J. Vac. Sci. Technol. A 7 (1989) 2906.

[18] H. Hölscher, U.D. Schwarz, R. Wiesendanger, Appl. Surf. Sci. 140 (1999) 344. 